Basrah Journal Of Surgery
Review Article

Bas J Surg, June, 26, 2020

\title{
IN COVID-19 PANDEMIC, A LITTLE NEGLECT MAY BREED GREAT MISCHIEF
}

\section{Ahmed M Al-Abbasi}

MB,ChB, FIBMS, FRCS, Professor of Otolaryngology, Department of Surgery, College of Medicine, University of Basrah, IRAQ.

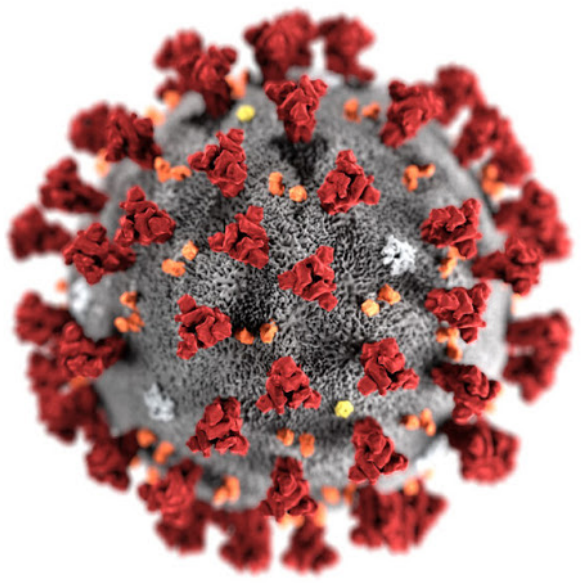

\begin{abstract}
COVID-19, the disease caused by SARS-CoV-2, is a highly communicable disease. There is urgent need for highlighting the ongoing outbreak to be public health emergency especially in our locality. Based on current published evidence, this review systematically summarizes the causative agent, epidemiology, presentation, diagnosis, treatment, prevention and surgeon precautions against COVID-19. Hopefully, this review may put a small stone for public health building in this regard and may afford a reference for future studies in our district.
\end{abstract}

\section{Overview}

Coronavirus disease 2019 (COVID-19) is a mild, moderate or severe respiratory tract infection caused by a newly evolving corona virus, SARS-CoV-2, that was first documented in Wuhan, China, in December $2019^{1}$.

While most people with COVID-19 develop mild or simple illness, around 14\% develop severe infection requiring hospitalization and oxygen therapy, and 5\% necessitate admission to an intensive care unit ${ }^{1}$. In severe illness, COVID-19 can be complicated by acute respiratory disease syndrome (ARDS), sepsis \& septic shock, and multi-organ failure including acute renal and cardiac failure ${ }^{2}$. Elderly and co-morbid disease have been blamed as risk factors for death ${ }^{2}$.

\section{Virology}

Corona viruses are positive-stranded RNA viruses with a crown-like shape under an electron microscope (coronam is the Latin term for crown) due to the presence of thorn glycoproteins on the envelope ${ }^{3}$.

Members of this large family of viruses can cause respiratory, enteric, hepatic, and neurological diseases in different animal species including; camels, cattle, cats, and bats. To date, seven human Corona viruses (HCoVs), capable of infecting humans, have been identified. In general, estimates suggest that $2 \%$ of the population are healthy 
carriers of Corona virus and that these viruses are responsible for about $5 \%$ to $10 \%$ of acute respiratory infections ${ }^{4}$.

The virus has round or elliptic and often pleomorphic form, and a diameter of approximately $60-140 \mathrm{~nm}$. Like other Corona viruses, it is sensitive to ultraviolet rays and heat. Furthermore, these viruses can be effectively inactivated by lipid solvents including; $75 \%$ ether, ethanol, chlorine-containing disinfectant, peroxyacetic acid and chloroform except for chlorhexidine ${ }^{4}$.

\section{Epidemiology}

Since December 2019, several cases having unexplainable pneumonia were consecutively reported in some hospitals in Wuhan city with a history of exposure to a large Hua'nan seafood market in Wuhan city, Hubei province, China. It has been confirmed to be an acute respiratory infection caused by a novel Corona virus. So far, the number of cases with no history of the Hua'nan seafood market exposure is increasing. In addition, clustered cases and confirmed cases without a history of travel to Wuhan emerged. Also, confirmed cases without clear exposure to the Wuhan seafood market have been found in many foreign countries or regions ${ }^{5}$. With close contacts to the confirmed patients who have been tracked, 583 people were released from medical observation that period, and 30,453 people were still undergoing medical observation ${ }^{6}$.

Wild animal, bat $^{7}$ is the most probable host of the 2019-Corona virus. It requires further verification whether pneumonia infection by the 2019-Corona virus is transmitted directly from bats or by an intermediate host.

Respiratory droplet spread is the main route of transmission, and it can also be transmitted through contact ${ }^{8}$. Although many details, such as the source of the virus and its ability to spread between people remain unknown, an increasing number of cases show the signs of human-to human transmission ${ }^{5,9}$. Till now there is no indication of viral mutation ${ }^{10}$.

The latency period is usually from 3 to 7 days, with a maximum of 14 days $^{7}$. Unlike SARSr- COVID-19 which is contagious during the latency period ${ }^{11}$.

The population in general are susceptible to the corona viral infection. The elderly and those with underlying diseases show more severe illness after infection. From modern information of the cases, most patients have a good prognosis, the illness of children is relatively mild, and a few patients are in serious condition. Death is more commonly seen in the elderly and those with chronic underlying diseases like diabetes, hypertension, and cardiovascular diseases ${ }^{8,12}$.

\section{Presentation and diagnosis}

The incubation period for COVID-19 extend to 14 days, with a median time of 4-5 days from exposure to the start of symptoms.

The signs and symptoms of COVID-19 at illness onset vary, but over the course of the disease, most persons with COVID-19 will experience the following ${ }^{13,14}$ : Fever (83$99 \%$ ), cough (59-82\%), fatigue (44-70\%), anorexia (40-84\%), shortness of breath (31$40 \%$ ), sputum production (28-33\%), and myalgia (11-35\%).

Atypical presentations have been described, and older adults and persons with medical co-morbidities may have delayed presentation of fever and respiratory symptoms ${ }^{15,16}$. In one study of 1,099 hospitalized patients, fever was present in only $44 \%$ at hospital admission but later developed in $89 \%$ during hospitalization ${ }^{13}$. Headache, confusion, rhinorrhea, sore throat, hemoptysis, vomiting, and diarrhea have been reported but are less common (less than 10\%) ) $^{13}$. Some persons with COVID-19 have experienced gastrointestinal symptoms such as diarrhea and nausea prior to developing fever and 
lower respiratory tract signs and symptoms. Anosmia or ageusia preceding the onset of respiratory symptoms has been anecdotally reported ${ }^{17}$.

The largest cohort of about 44,000 persons with COVID-19 from China showed that illness severity can range from mild to critical ${ }^{18}$ : Mild to moderate (mild symptoms up to mild pneumonia qual to $81 \%$ ), Severe (dyspnea, hypoxia, or $>50 \%$ lung involvement on imaging equal to $14 \%$ ), and Critical (respiratory failure, shock, or multiorgan system dysfunction equal to $5 \%$ ).

All deaths in this study occurred amongst patients with critical illness and the overall case mortality rate was $2.3 \%{ }^{18}$. The mortality rate among patients with critical disease was $49 \%{ }^{18}$. Among children in China, illness severity was lower with $94 \%$ having asymptomatic, mild or moderate disease, $5 \%$ having severe disease, and $<1 \%$ having critical disease ${ }^{19}$.

Age is a strong risk factor for severe illness, complications, and death ${ }^{13,16}$. Among more than 44,000 confirmed cases of COVID-19 in China, the case fatality rate was highest among older persons ( 80 years and above $)^{20}$.

Patients in China with no reported underlying medical conditions had an overall case fatality of $0.9 \%$, but case fatality was higher in patients with comorbidities like: cardiovascular disease, diabetes, chronic respiratory disease, hypertension, and cancer ${ }^{21}$. Heart disease, hypertension, prior stroke, diabetes, chronic lung disease, and chronic kidney disease have all been associated with increased illness severity and adverse outcomes ${ }^{13}$.

There are no data concerning the possibility of re-infection with SARS-CoV-2 after recovery from COVID-19. Viral RNA shedding declines with resolution of symptoms, and may continue for days to weeks ${ }^{22,23}$. However, the detection of RNA during convalescence does not necessarily indicate the presence of viable infectious virus. Clinical recovery has been correlated with the detection of $\operatorname{IgM}$ and $\operatorname{IgG}$ antibodies which signal the development of immunity ${ }^{24}$.

\section{Investigations}

Viral Testing: Diagnosis of COVID-19 requires finding of SARS-CoV-2 RNA by reverse transcription polymerase chain reaction (RT-PCR). Detection of SARS-CoV-2 viral RNA is better in nasopharynx samples compared to throat samples ${ }^{22}$. Recognition of SARS-CoV-2 RNA in blood may be a marker of severe illness ${ }^{25}$.

Laboratory Findings: Lymphopenia is the most common lab finding in COVID-19 and is found in as many as $83 \%$ of hospitalized patients ${ }^{13}$. Lymphopenia, neutrophilia, elevated serum alanine aminotransferase and aspartate aminotransferase levels, elevated lactate dehydrogenase, high CRP, and high ferritin levels may be associated with greater illness severity13. Elevated D-dimer and lymphopenia have been associated with mortality. Procalcitonin is typically normal on admission, but may increase among those admitted to the $\mathrm{ICU}^{14}$. High plasma levels of inflammatory makers usually seen in patients with critical illness, signifying potential immune dysregulation ${ }^{25}$.

Radiographic Findings: Chest radiographs of patients with COVID-19 typically demonstrate bilateral air-space consolidation, though patients may have unremarkable chest radiographs early in the disease ${ }^{13}$. Chest CT images from patients with COVID-19 typically demonstrate bilateral, peripheral ground glass opacities ${ }^{26}$. Because this chest CT imaging pattern is non-specific and overlaps with other infections, the diagnostic value of chest CT imaging for COVID-19 may be low and dependent upon radiographic interpretation $^{27}$. One study found that $56 \%$ of patients who presented within 2 days of diagnosis had a normal $\mathrm{CT}^{28}$. Conversely, other studies have also identified chest CT abnormalities in patients prior to the detection of SARS-CoV-2 RNA ${ }^{29}$. Given the 
variability in chest imaging findings, chest radiograph or $\mathrm{CT}$ alone is not recommended for the diagnosis of COVID-19. The American College of Radiology also does not recommend CT for screening or as a first-line test for diagnosis of COVID- $19^{30}$.

\section{Treatment}

Mild cases: emphasizing on diminish transmission. This can be done at home if the hospitals become crowded or in hospital, if there are just few patients or small clusters. Supply patient with mild COVID-19 with symptomatic treatment such as antipyretics for fever. Advise patients with mild COVID-19 about signs and symptoms of complicated disease, if they develop any of these symptoms, they should ask for urgent care.

Sever cases: Patients with emergency signs (obstructed or absent breathing, severe respiratory distress, central cyanosis, shock, coma or convulsions) should get airway management and oxygen therapy during resuscitation. Closely observe patients with COVID-19 for signs of clinical worsening, such as quickly progressive respiratory failure and sepsis. Respond immediately with supportive care interventions.

Giving empiric antimicrobials to treat all likely pathogens causing severe respiratory infection and sepsis should be as soon as possible within 1 hour.

Patients may continue to have increased work of breathing or hypoxemia even when oxygen is delivered via a face mask with reservoir bag. Hypoxemic respiratory failure in ARDS commonly results from intrapulmonary ventilation-perfusion mismatch or shunt and usually need mechanical ventilation ${ }^{31}$.

In cases of septic shock, standard care includes early recognition and treatments within 1 hour of recognition like antimicrobial therapy, initiation of fluid bolus, and vasopressors for hypotension ${ }^{31}$. The use of central venous and arterial catheters should be based on resource availability and individual patient needs ${ }^{32,33}$.

\section{Surgeon's precautions}

There should be a universal transport practice for bringing COVID-19 patient to the hospital to reduce the exposure to the paramedic staff and the surgical team. There should be an operation theatre (OT) enthusiastic only for COVID-19 cases, situated in a corner of the hospital complex ${ }^{34}$.

Traffic and flow of contaminated air should be minimized by locking all doors of the OT during surgery, with only one possible route for entry/exit through the scrub room. During the procedure, a runner is stationed outside the OT if additional drugs or equipment are needed.

No unnecessary movement allowed inside the OT. All single-use equipment (even unopened) in the room should be discarded after the operation. Therefore, it is necessary that what is vital in the surgical procedure should only be displayed. There should be a 30-min interval between the consecutive cases in the same OT $^{35}$. All electronic gadgets like pagers, laptop or mobile and hospital case sheets should be left outside the OT. Disposable pens are to be used. Donning and doffing of personal protective equipment (PPE) should be done under supervision and with extreme care. Standard PPE includes a face shield, mask, waterproof gown, double gloves and shoe covers. There is an argument whether N 95 mask or a ordinary surgical mask need to be used. Informed consent and charting intraoperative period are done electronically. In patients proved to have COVID-19 who need urgent surgery, the risk and benefit of going on or canceling need to be weighted.

Anaesthesia machine should be covered with a plastic wrap. The number of staff in and out of the OT should be limited. In COVID-19 patients, regional anaesthesia is 
favorable over general anaesthesia. Before the start of each operation, the anaesthesiologist puts all the drugs and equipment required for the operation into a tray to avoid handling of the drug trolley during the case. After the surgery, the anaesthetic breathing circuit and the container of soda lime are discarded without delay. All the surfaces of medical devices are cleaned with quaternary ammonium chloride disinfectant. The OT is cleaned with sodium hypochlorite and treated with hydrogen peroxide vaporization or ultraviolet $\mathrm{C}$ irradiation. During laparoscopy, $\mathrm{CO}_{2}$ insufflations and the use of diathermy or harmonic scalpel generate significant aerosols which may be released when pnemo-peritoneum is released. Therefore, laparoscopic procedures need to be avoided in COVID-19 patients. The power setting of electrocautery should be as low as possible. Avoid coagulation of the same spot for a long time to reduce the surgical smoke. Spanish Association of Coloproctology suggested intra-corporeal over extracorporeal anastomosis to avoid contamination with a fecal aerosol. Liberal use of suction device to remove smoke and aerosol during operation is a must especially before converting laparoscopy to open procedure ${ }^{36}$.

No visitors or accompanying persons are allowed in the hospital and all the shop, restaurant and facilities should remain closed ${ }^{36}$. Telemedicine consultation is recommended for the patient postoperatively with the surgeon in order to reduce the hospital visits. WhatsApp or mobile consultation are more feasible ${ }^{36,37}$. To keep surgeons family safe, the government may make hotel accommodations available for healthcare workers who cannot or prefer not to go home following patient care activities.

Alertness to the fact that viral contamination of surfaces is a known mean of spread of infection $^{37}$, therefore keeping hand sanitizer and/or disposable gloves for use to touch and handling any object is mandatory. Cleaning of cell phone frequently before, during, and after patient care activities ${ }^{38}$. Cell phones may be kept in a Ziploc bag during work activities. The phone can be used while in the bag. Consider removing clothes and washing them upon arrival home, reducing physical contact with family members and wash hands frequently. Cleaning of hard surfaces at home with an effective disinfectant solution (e.g. $60 \%$ alcohol) ${ }^{39}$.

\section{Conclusion}

COVID-19, although sometimes simple, but it is not negligible. It is like a wild animal that could leave you but might kill you. Bats are likely an important reservoir for the virus. The main mode of transmission of the virus is through inhalation of respiratory droplets and indirect or direct contact. Incubation period extends to 14 days, with a median time of 4-5 days from exposure to the start of symptoms. The risk of getting the infection and increase severity occur in older age and concomitant comorbidities. Till now, no specific drug or vaccine is available. This pandemic has shown the unpreparedness of health systems even in developed countries for such battle.

There are a lot of information about this world disaster and the ways of prevention, spread, communicability, diagnosis, treatment, whether it has an end or not, will it return again in the future, and the material and moral effects after its end. All these questions still are not fully clarified and answered for now.

\section{References}

1. Team NCPERE. Vital surveillances: The epidemiological characteristics of an outbreak of 2019 novel coronavirus diseases (COVID-19)-China. China CDC Weekly. 2020;2(8):113-22.

2. Yang X, Yu Y, Xu J, Shu H, Xia J, Liu H et al. Clinical course and outcomes of critically ill patients with SARS-CoV-2 pneumonia in Wuhan, China: a single-centered, retrospective, observational study. Lancet Respir Med. 2020

3.Chan JF, To KK, Tse H, Jin DY, Yuen KY. Interspecies transmission and emergence of novel viruses: lessons from bats and birds. Trends Microbiol. 2013 Oct;21(10):544-55. 
4.Chen Y, Liu Q, Guo D. Emerging coronaviruses: Genome structure, replication, and pathogenesis. J. Med. Virol. 2020 Apr;92(4):418-423.

5. Zhu N, Zhang D, Wang W, et al. A novel coronavirus from patientswith pneumonia in China, 2019. N Engl J Med. 2020;382(8):727-733

6. Health Emergency Office of National Health Commission of the PRC. Update on the epidemic of novel coronavirus (2019-nCoV) infected pneumonia as at $24: 00$ on 26 January.http:/www.nhc.gov.cn/xcs/yqfkdt/2 02001/3882fdcdbfdc4b4fa4e3a829b62d518e. shtml. Accessed 26 Jan 2020

7. Zhou P, Yang XL, Wang XG, Hu B, Zhang L, Zhang W, et al. A pneumonia outbreak associated with a new coronavirus of probable bat origin. Nature. 2020.

8. General Office of National Health Committee. Office of State Administration of Traditional Chinese Medicine. Notice on the issuance of a programme for the diagnosis and treatment of novel coronavirus (2019-nCoV) infected pneumonia (Trial Version 4). 2020. http://bgs.satcm.gov.cn/ zhengcewenjian/2020-01-28/12576.html. Accessed 29 Jan 2020.

9. Zhu N, Zhang D, Wang W, Li X, Yang B, Song J, et al. A novel coronavirus from patients with pneumonia in China, 2019. N Engl J Med. 2020.

10. General Office of National Health Committee. Office of State Administration of Traditional Chinese Medicine. Notice on the issuance of a program for the diagnosis and treatment of novel coronavirus (2019-nCoV) infected pneumonia (Trial Version 3). 2020.http://www.nhc.gov.cn/xcs/zhengcwj/202001/f492c9153ea9437bb587ce2ffcbee1fa.shtml. Accessed 24 Jan 2020.

11. Information Office of the State Council. Information office to hold press conference on joint preventing and controlling the epidemic of novel coronavirus (2019-nCoV) infected pneumonia. 2020. http://www.scio.gov.cn/ xwfbh/xwbfbh/wqfbh/42311/42478/index.htm. Accessed 26 Jan 2020.

12. Huang C, Wang Y, Li X, Ren L, Zhao J, Hu Y, et al. Clinical features of patients infected with 2019 novel coronavirus in Wuhan, China. Lancet. 2020

13.Guan WJ, Ni ZY, Hu Y, et al. Clinical Characteristics of Coronavirus Disease 2019 in China. N Engl J Med 2020;382:1708-20.

14.Chen N, Zhou M, Dong X, et al. Epidemiological and clinical characteristics of 99 cases of 2019 novel coronavirus pneumonia in Wuhan, China: a descriptive study. Lancet 2020;395:507-13.

15.Zhou F, Yu T, Du R, et al. Clinical course and risk factors for mortality of adult inpatients with COVID-19 in Wuhan, China: a retrospective cohort study. Lancet 2020.

16. Yang X, Yu Y, Xu J, et al. Clinical course and outcomes of critically ill patients with SARS-CoV-2 pneumonia in Wuhan, China: a single-centered, retrospective, observational study. Lancet Respir Med 2020.

17.Giacomelli A, Pezzati L, Conti F, et al. Self-reported olfactory and taste disorders in SARS-CoV-2 patients: a cross-sectional study. Clin Infect Dis 2020

18. Wu Z, McGoogan JM. Characteristics of and Important Lessons From the Coronavirus Disease 2019 (COVID-19) Outbreak in China: Summary of a Report of 72314 Cases From the Chinese Center for Disease Control and Prevention. JAMA 2020.

19.Dong Y, Mo X, Hu Y, et al. Epidemiology of COVID-19 Among Children in China. Pediatrics 2020.

20. Team CC-R. Severe Outcomes Among Patients with Coronavirus Disease 2019 (COVID-19) - United States, February 12March 16, 2020. MMWR Morb Mortal Wkly Rep 2020;69:343-6.

21. Novel Coronavirus Pneumonia Emergency Response Epidemiology T. [The epidemiological characteristics of an outbreak of 2019 novel coronavirus diseases (COVID-19) in China]. Zhonghua Liu Xing Bing Xue Za Zhi 2020;41:145-51.

22. Zou L, Ruan F, Huang M, et al. SARS-CoV-2 Viral Load in Upper Respiratory Specimens of Infected Patients. N Engl J Med 2020;382:1177-9.

23. Young BE, Ong SWX, Kalimuddin S, et al. Epidemiologic Features and Clinical Course of Patients Infected With SARS-CoV-2 in Singapore. in 2020.

24. To KK, Tsang OT, Leung WS, et al. Temporal profiles of viral load in posterior oropharyngeal saliva samples and serum antibody responses during infection by SARS-CoV-2: an observational cohort study. Lancet Infect Dis 2020.

25. Qin C, Zhou L, Hu Z, et al. Dysregulation of immune response in patients with COVID-19 in Wuhan, China. Clin Infect Dis 2020 .

26.Wu C, Chen X, Cai Y, et al. Risk Factors Associated With Acute Respiratory Distress Syndrome and Death in Patients With Coronavirus Disease 2019 Pneumonia in Wuhan, China. JAMA Intern Med 2020.

27.Ai T, Yang Z, Hou H, et al. Correlation of Chest CT and RT-PCR Testing in Coronavirus Disease 2019 (COVID-19) in China: A Report of 1014 Cases. Radiology 2020:200642.

28. Bernheim A, Mei X, Huang M, et al. Chest CT Findings in Coronavirus Disease-19 (COVID-19): Relationship to Duration of Infection. Radiology 2020:200463.

29. Shi H, Han X, Jiang N, et al. Radiological findings from 81 patients with COVID-19 pneumonia in Wuhan, China: a descriptive study. Lancet Infect Dis 2020;20:425-34.

30. American College of Radiology. ACR recommendations for the use of chest radiography and computed tomography (CT) for suspected COVID-19 infection. Accessed at www.acr.org/Advocacy-and-Economics/ACR-Position-Statements/ Recommendationsfor-Chest-Radiography-and-CT-for-Suspected-COVID19-Infection on 5 April 2020.

31. Rhodes A, Evans LE, Alhazzani W, Levy MM, Antonelli M, Ferrer R et al. Surviving Sepsis Campaign: International Guidelines for Management of Sepsis and Septic Shock: 2016. Intensive Care Med. 2017;43(3):304-77. Epub 2017/01/20. doi: 10.1007/s00134-017-4683-6. PubMed PMID: 28101605.

32. Weiss SL, Peters MJ, Alhazzani W, Agus MSD, Flori HR, Inwald DP et al. Surviving Sepsis Campaign International Guidelines for the Management of Septic Shock and Sepsis-Associated Organ Dysfunction in Children. Pediatr Crit Care Med. 2020;21(2):e52e106. Epub 2020/02/08.

33. WHO. Pocket book of hospital care for children: guidelines for the management of common childhood illnesses. Geneva: World Health Organization; 2013 (http://www.who.int/maternal_child_adolescent/documents/child_hospital_care/en/, accessed 4 March 2020).

34. Spinelli A, Pellino G (2020) Covid-19 pandemic: perspective on an unfolding crisis. Br J Surg.

35. Ti LK, Ang LS, Foong TW (2020) What we do when a COVID-19 patient needs an operation: operating roompreparation and guidance. Can J Anesth:1-3.

36. Pellino G, Spinelli A (2020) How COVID-19 outbreak is impacting colorectal cancer patients in Italy: a long shadow beyond infection. Dis Colon Rectum.

37. Ferioli M, Cisternino C, Leo V, Pisani L, Palange P, Nava S. Protecting healthcare workers from SARS-CoV-2 infection: practical indications. Eur Respir Rev. 2020;29(155):200068. Published 2020 Apr 3.

38. Lurie N, et al. Developing Covid-19 Vaccines at Pandemic Speed. NEJM. March 30, 2020

39. American College of Radiology .COVID 19: Considerations for Optimum Surgeon Protection Before, During, and After Operation. Accessed at www.facs.org/covid-19/clinical-guidance/surgeon. 